\title{
Global Kidney Health Atlas: a spotlight on the Asia- Pacific sector
}

\author{
Joyita Bharati ${ }^{1}$, Vivekanand Jha ${ }^{2,3,4}$ \\ ${ }^{1}$ Department of Nephrology, Postgraduate Institute of Medical Education and Research, Chandigarh, India \\ ${ }^{2}$ The George Institute for Global Health, New Delhi, India \\ ${ }^{3}$ School of Public Health, Imperial College, London, United Kingdom \\ ${ }^{4}$ Prasanna School of Public Health, Manipal Academy of Higher Education, Manipal, India
}

Chronic kidney disease (CKD) is a unique public health problem in terms of high expenses required for its management and its increasing worldwide incidence. Understanding the existing structure of CKD treatment and its epidemiology is pivotal for equitable kidney care globally. The Global Kidney Health Atlas (GKHA) was launched by the International Society of Nephrology (ISN) in 2017 as a part of its "Bridging the Gaps" strategy with a vision to understand the global kidney care capacity. Two rounds of GKHA surveys were conducted and published in 2017 and 2019, respectively. The GKHA surveys showed significant variability in kidney care among global regions. Asia is the largest of all continents and distinct for its heterogeneity. The Asia-Pacific sector comprises four main regional constructs of the ISN; North and East Asia, Oceania and South East Asia, South Asia, and the Middle East. Kidney care capacity varies among these regions in terms of government spending, epidemiology of kidney diseases, and workforce availability. In this narrative review, we highlight the differences in kidney care delivery among the regions of the Asia-Pacific sector based on information from GKHA and emphasize the priority areas on which stakeholders should concentrate their efforts.

Keywords: Asia, Chronic kidney disease, Global goals, Global Kidney Health Atlas

\section{Introduction}

Even small decrements in kidney function, determined by a reduction in the glomerular filtration rate and/or an increase in urinary protein excretion, have serious adverse health consequences. Advances in our understanding of this concept have led to concurrent changes in our appreciation of chronic kidney disease (CKD) as a global public health burden. These advances in understanding were fa- cilitated by development of a globally accepted framework for diagnosis and classification of CKD and adoption of an overall health-risk approach to CKD. This approach has replaced the long-standing perspective of singular focus on kidney failure as the main adverse consequence of CKD.

Over the last 20 years, several projects, particularly the Global Burden of Disease Study and CKD Prognosis Consortium, have provided compelling data on the CKD disease burden and its adverse health consequences. For example,

Received: October 20, 2021; Revised: November 15, 2021; Accepted: November 15, 2021

Correspondence: Vivekanand Jha

The George Institute for Global Health, 308-09, Third Floor, Elegance Tower, Plot. No. 8, Jasola District Centre, New Delhi 110025, India.

E-mail:vjha@georgrinstitute.org.in

ORCID: https://orcid.org/0000-0002-8015-9470

Copyright (C) 2022 by The Korean Society of Nephrology

(a) This is an Open Access article distributed under the terms of the Creative Commons Attribution Non-Commercial and No Derivatives License (http:// creativecommons.org/licenses/by-nc-nd/4.0/) which permits unrestricted non-commercial use, distribution of the material without any modifications, and reproduction in any medium, provided the original works properly cited. 
CKD is the third fastest-growing cause of death worldwide and is projected to be the fifth leading cause of death by 2040. This projection is alarming because CKD is currently the 16th leading cause of death. Approximately 850 million people are living with some form of kidney disease, almost double the number of individuals with diabetes.

Treatment of advanced CKD with dialysis and/or kidney transplantation is expensive, both for health systems and individuals. According to a 2015 systematic review, less than half of all people needing kidney replacement therapy (KRT) had access to this therapy [1]. Less than $1 \%$ of all incident dialysis patients in Sub-Saharan Africa were still on dialysis after 1 year [2]. Development of sustainable and scalable strategies to reduce the CKD burden is needed urgently when considering the simultaneously increasing burden of chronic diseases such as diabetes mellitus, hypertension, and metabolic syndrome. These burdens are exacerbated by global population aging and emergence of new risk factors. These risk factors include environmental changes such as warming climates and increasing air pollution.

The realization of the urgency of the situation was accompanied by the realization of a data deficiency. This lack of data was related to various aspects of kidney care, particularly the resources available in various parts of the world to respond to this emerging public health threat, and constituted a major barrier to our ability to best address this burgeoning problem in all countries.

The International Society of Nephrology (ISN) is a global organization of renal health professionals with a vision to strive for "a future where all people have equitable access to sustainable kidney health" [3]. In order to achieve this vision, the ISN attempts to bridge the gaps of knowledge, policy, and care; improve human resource provision; increase the knowledge base through expanded research; and connect communities through its global convening power, education, and advocacy. The Global Kidney Health Atlas (GKHA) initiative was launched as part of this 'Bridging the Gaps' strategy in 2017 to assimilate available global kidney care capacity data with a vision to advocate for increased access to kidney disease care worldwide.

The GKHA study measures the current standards of and resources available for kidney healthcare delivery across countries, with a focus on disease burden and economics. Electronic questionnaire-based surveys among opinion leaders and literature reviews are the two pillars of the
GKHA. The aim is to gather and compare data concerning the capacity to deliver optimal kidney care among and within countries. The GKHA survey uses the six domains of universal health coverage advocated by the World Health Organization (WHO), i.e., information system, services delivery, workforce, financing, access to essential medicines, and leadership governance, to measure performance of health systems (Fig. 1) [4]. Countries are categorized based on World Bank income group and ISN region [3]. Two survey cycles were conducted, one in 2017 and the other in 2019; both resulted in high-impact publications. Future surveys are planned for once every 4 years. Following the 2019 survey, a series of independent publications addressing global variations according to WHO health system domain and ISN region was published. These surveys have demonstrated the substantial variability among countries in kidney care capacity in terms of public funding, workforce, service availability, and existence of data collection platforms (registries). As expected, the gaps in capacity, reach, and quality of care are greater in low and low-middle-income countries, especially those in Asia and Africa [5]. Given that most such countries lack national and/or regional registries, data from the GKHA surveys are the only source of information.

By providing a snapshot of the latest indicators of kidney care capacity, the GKHA provides an important platform for policymakers to identify gaps in kidney healthcare delivery. The response to the 2018 survey by 160 countries representing over $98 \%$ of the world's population reflects global willingness for collaboration in confronting kidney care issues. GKHA also serves as a tool to monitor progress in global renal care capacity in a systematic manner. The two GKHA surveys published in 2017 and 2019 resulted in positive changes in a global kidney care capacity. The number of national registries on CKD, acute kidney injury, dialysis, and transplant has increased; and more countries are acquiring the ability to offer peritoneal dialysis (PD) as a means of chronic dialysis [4]. The GKHA 2019 survey focused on the care of patients with kidney failure, which included KRT and conservative care, in addition to other aspects addressed by the GKHA 2017 survey. While hemodialysis (HD) is available in all countries, only one-quarter of low-income countries have facilities to provide PD or kidney transplantation. Among the low-income countries, less than $10 \%$ of patients are able to access dialysis services. Similarly, surveillance systems are scarce in low-income countries; 


\section{Global Kidney Health Atlas indicators of kidney care capacity}
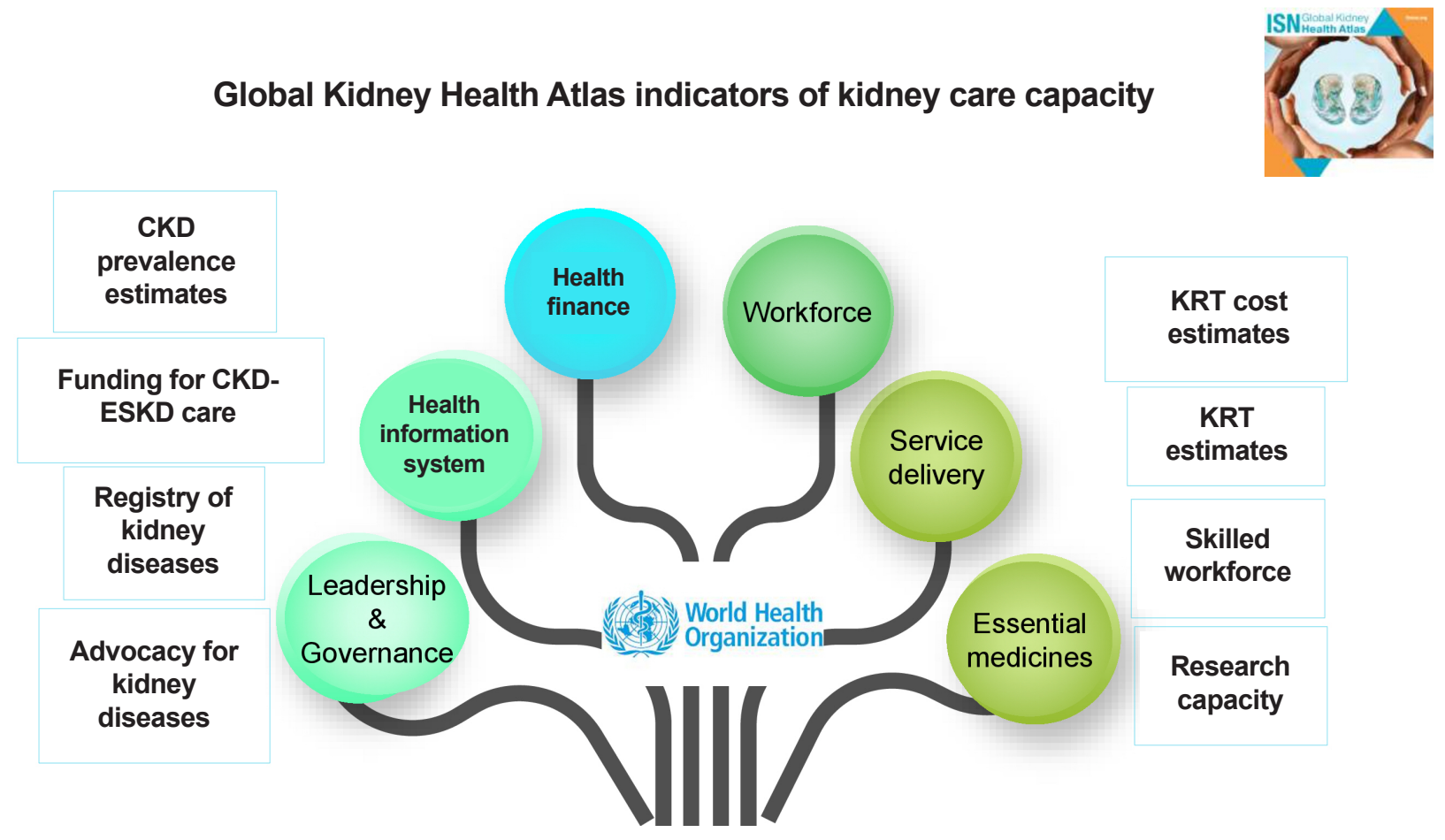

Figure 1. Global Kidney Health Atlas survey assessment of kidney care capacity of coun-tries/jurisdictions across the globe is based on the World Health Organization's six dimen-sions of health system performance evaluation.

CKD, chronic kidney disease; ESKD, end-stage kidney disease; KRT, kidney replacement therapy.

Rwanda is the only such country with data on treated kidney failure [2]. Economic development and public awareness are important obstacles in accessing end-stage kidney disease (ESKD) care in low-income countries. GKHA has proposed country-level scorecards, which allows stakeholders, especially in low-income countries, to identify priority areas on which to concentrate and allocate resources. For global and multilateral organizations, the GKHA provides a platform for comparison and collaboration and an impetus toward working for global goals. In addition to accessibility of renal care, GKHA addresses the quality of care and equity of healthcare access.

Asia is the largest continent, with a population of approximately 4.7 billion. Asia is diverse in terms of wealth, climate, culture, socioeconomic and development indicators, and healthcare delivery systems. In this narrative review, we discuss the status of kidney care capacity and the gaps in kidney care delivery in Asia to highlight the variability among regions. A summary of the major parameters of kidney care capacity in the different ISN regions of the Asia-Pacific sector is shown in Table 1.

\section{Global Kidney Health Atlas: a spotlight on Asia}

Four of the 10 world ISN regions are located in the Asia-Pacific sector, i.e., North and East Asia, Oceania and South East Asia (OSEA), South Asia, and the Middle East (Fig. 2). Some countries in newly independent states and Russia are regions also geographically included in Asia; however, these are not covered in this review.

Many countries in the region have inadequate kidney health care capacity in terms of availability of KRT (Fig. 3), low public funding or access to KRT, high disease burden, and poor research capacity [6]. The epidemiology of kidney disease varies in the tropical Asian region from that of western countries. Furthermore, Asia is expected to have the highest growth in KRT use by 2030 [1]. Indeed, between 2003 and 2016, incidence rates of KRT have significantly increased in the growing economies of East and Southeast Asia [7].

Disparity in access to healthcare, particularly KRT, has been observed among women and children in low- and low-middle-income countries in Asia [8]. The gaps in meet- 

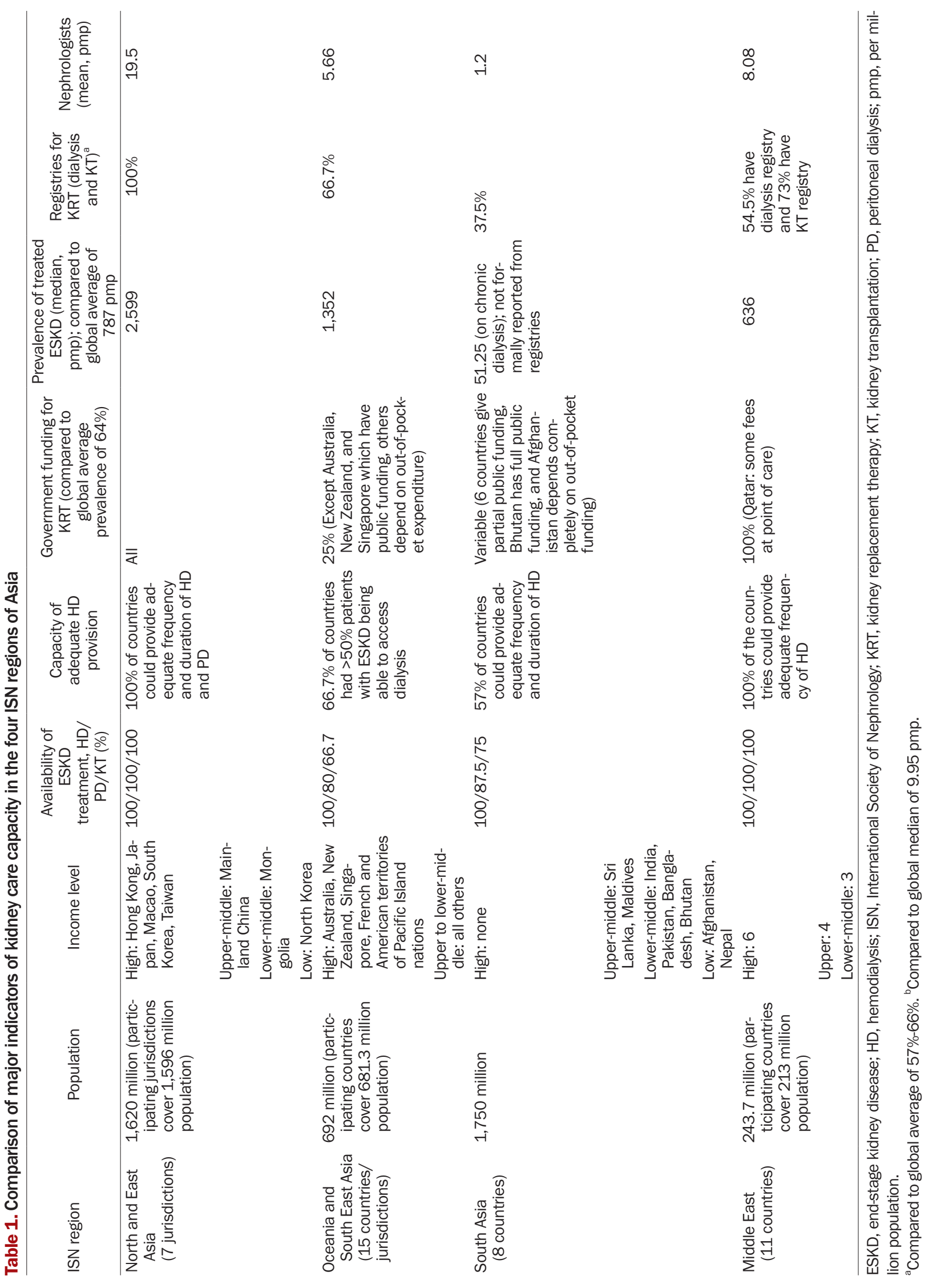


\section{ISN construct of Asia}

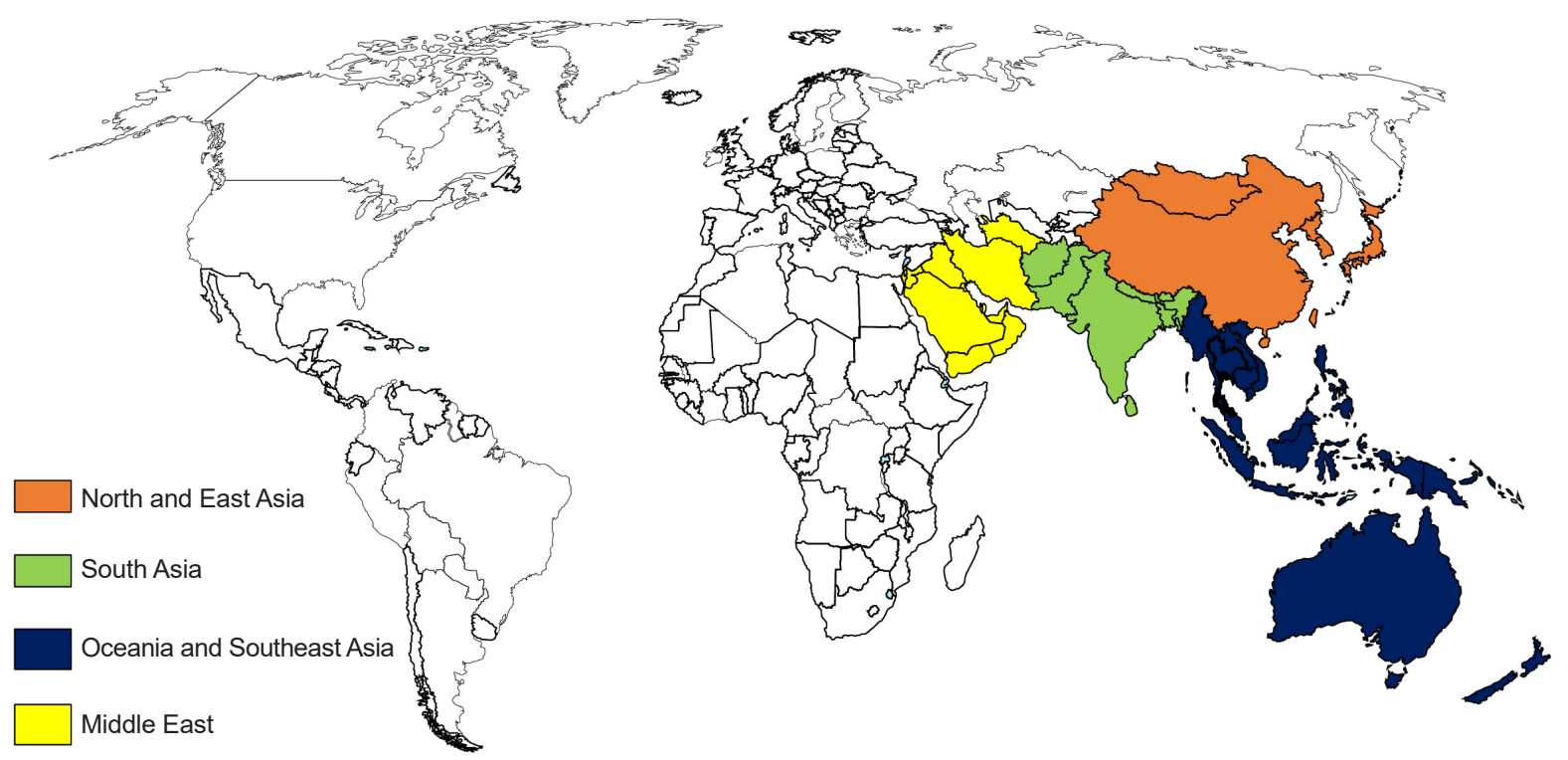

Figure 2. International Society of Nephrology (ISN) construct of Asia comprises four regions predom-inantly; North and East Asia, Oceania and South East Asia, South Asia, and the Middle East.

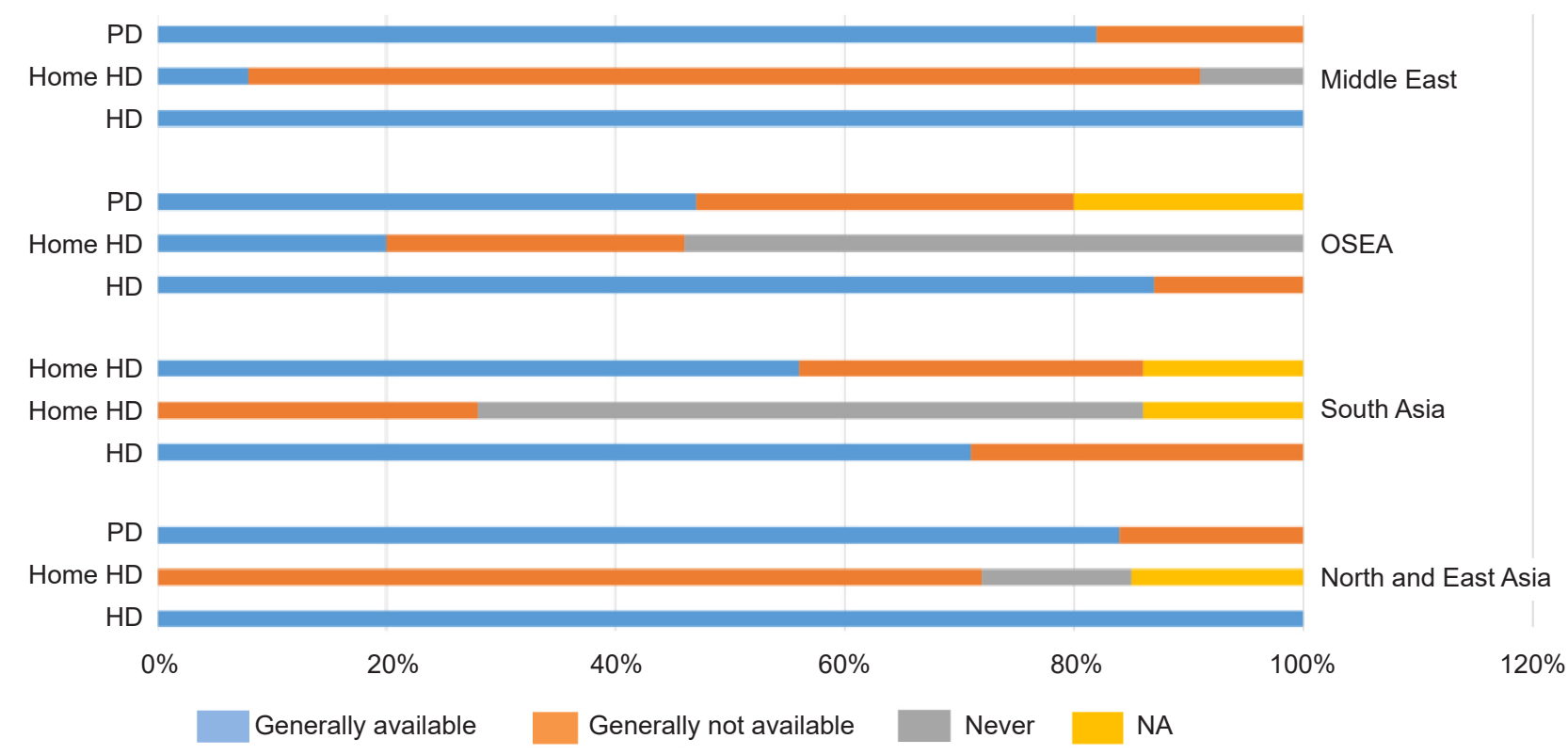

Figure 3. Availability of various modalities of kidney replacement therapy for patients with kidney failure across the Asia-Pacific sector.

PD, peritoneal dialysis; HD, hemodialysis; OSEA, Oceania and South East Asia; NA, not available. 
ing the needs of Asians with kidney disease would be best addressed by focusing on distinct strategies based on individual country needs. For example, South Asian countries maintaining hotspots of CKD of unknown etiology [9] must focus on identifying causative factors and developing preventive strategies that target occurrence and progression of CKD. Similarly, with the rising epidemic of metabolic syndrome in the Asia-Pacific sector [10], effective preventive strategies are imperative at all levels in all regions. The incidence of diabetes mellitus, the most common cause of CKD worldwide, is increasing rapidly in the sector. Therefore, public and government awareness concerning renal disease trends is warranted. By providing relevant kidney health information, GKHA is likely to positively influence health policy decisions in the low-income countries in Asia.

\section{North and East Asia}

North and East Asia includes eight jurisdictions, China, Japan, Hong Kong, Taiwan, Mongolia, North Korea, South Korea, and Japan. Other than China and Mongolia, which are upper-middle and lower-middle-class countries, respectively, these jurisdictions are classified as high income. Government expenditure on health comprises $~ 50 \%$ to $60 \%$ of total health expenditure, with Japan spending $10.9 \%$ of the gross domestic product on health [11]. Taiwan has the highest global prevalence of treated ESKD. Among a total of 108,873 patients receiving KRT in South Korea, 81,760 (75.1\%) were receiving HD; 5,960 (5.5\%) PD; and 21,153 (19.4\%) kidney transplantation as of 2019 [12]. Because countries in this region have a large aging population and have already transitioned from glomerulonephritis to high burden of metabolic diseases [13,14], they are likely to have increasing incidence of ESKD. Additionally, use of PD, which needs fewer resources, can help bridge the gap in treatment of ESKD in rural areas of developing economies in this region. Until further data are provided by the China Kidney Disease Network (CK-NET), exact prevalence and incidence of treated ESKD in mainland China are unknown. Japan and Taiwan rely on HD for treating ESKD ( $>95 \%$ of patients with ESKD), but Hong Kong provides PD more frequently than HD. All jurisdictions except Hong Kong have access to government support for KRT, and $71.4 \%$ of these countries provide public funding for nondialysis medications. Patients are not required to pay fees in most of these jurisdictions. For example, in mainland China, $76.6 \%$ of the dialysis costs were covered by the government as of 2015 [15]. Kidney transplantation is available in all jurisdictions, and all had a national waiting list and standard framework for organ procurement. CKD and ESKD are identified as health priorities in all jurisdictions.

\section{South Asia}

Eight countries, India, Bangladesh, Pakistan, Afghanistan, Bhutan, Nepal, Sri Lanka, and the Maldives, comprise the South Asia region and are in the World Bank's low, lower-middle, and upper-middle-income categories. This region is densely populated with a huge, but inadequately reported, CKD burden and immature healthcare systems. The main causes of CKD include infections; low birth weight due to maternal malnutrition and environmental factors; and diseases such as diabetes mellitus, hypertension, and glomerulonephritis. The incidence of diabetes mellitus, hypertension, and obesity has increased significantly due to sedentary lifestyles and poor dietary habits in these predominantly agrarian communities. Therefore, preventive interventions to reduce kidney disease in patients with diabetes and hypertension are likely to be cost-effective. There is no regular reporting of incidences of kidney disease or dialysis in this region. A large part of the weak kidney care capacity of South Asian countries arises from low economic development and low government spending on health $(\sim 4 \%$ of gross domestic product). Governmental lack of awareness contributes to the general lack of awareness and dissemination of misinformation concerning kidney diseases. This amplifies the CKD burden.

In all these countries, HD is more widely used than PD; the prevalence of $\mathrm{HD}$ use is 26.15 per million population compared to 1.65 per million population for PD use [16]. Also, the annual cost of PD (USD [\$] 8,764) is more than the annual cost of HD $(\$ 5,202)$ in most countries in South Asia. Promotion of PD with locally produced dialysis fluid, simplifying HD machine technology, and using telemedicine to manage integrated care of ESKD in patients located in remote places are some innovative ways to increase treatment accessibility. Collaboration with international professional organizations and industry partnership would lower dialysis and kidney transplantation costs for patients in low-income South Asian countries. Training the workforce in in- 
terventions for vascular access, immunological procedures such as human leukocyte antigen typing, and PD delivery would help close the gap in KRT delivery. Safeguarding the nephrology workforce in low-income South Asian countries is crucial to meet the challenge of a CKD epidemic. The incidence of ESKD in these low-income countries is increasing, and a large number of patients are likely to be unable to receive KRT [1].

\section{Oceania and South East Asia}

Of the total 30 countries listed in the ISN construct of OSEA, 15 participated in the GKHA survey. In addition to Australia, New Zealand, and South East Asian countries, the Pacific Island nations include French and American territories. This region is the most diverse of all in terms of culture, ethnicity, language, income level, healthcare expenditure, and population density [17]. Public spending on health expenditure is variable, with high-income countries investing more ( $9 \%$ of gross domestic product) on healthcare.

There has been a rapid growth in the incidence of treated kidney failure in the region, particularly in Singapore, Thailand, and Brunei Darussalam, partly due to the rapidly aging population and increased prevalence of metabolic diseases. KRT is not widely accessible in low-middle income countries of the Pacific Islands as well as a few South East Asian countries. However, high-income countries, comprising $60 \%$ of those in the OSEA region, provide high-quality, publicly-funded KRT. The cost of PD is lower in the OSEA region, except in Myanmar and Brunei Darussalam, than in other global regions (mean annual costs of $\$ 16,479 \mathrm{vs.} \mathrm{glob-}$ al mean annual costs of $\$ 20,524$ ). Thailand is exemplary in performance of PD as a widely used KRT; a PD-first policy was initiated in Thailand after careful cost analysis studies. Thailand has the highest incidence of treated ESKD in the world (299 per million population). Similarly, the neighboring countries of the Philippines and Malaysia have reported large increases in the incidence of treated ESKD over the last few decades despite limited public funding for KRT [18]. Most of the increase in the incidence of ESKD has been attributed to diabetes mellitus.

Kidney transplantation is available in two-thirds of the OSEA countries. Myanmar, Indonesia, and Brunei Darussalam have only living donor kidney transplant programs. Most countries require patients to pay between $1 \%$ and $50 \%$ of the costs of kidney transplantation; in Australia, however, transplantation is publicly funded.

Australia is the only country in the OSEA region without a shortage of kidney care providers. The Pacific Islands nations and some lower-middle-income South East Asian countries have limited KRT capacity. These countries have not yet prioritized kidney health at the national level even though CKD has a significant negative impact on death rates and disability-adjusted life years [19]. Cambodia has recently been able to establish KRT facilities with the help of multilateral partnership and collaboration with organizations (ISN) and neighboring countries (Japan) [17]. There are two unique challenges to be confronted in the OSEA region in terms of kidney care capacity. First, the indigenous communities are shown to have a $30 \%$ to $40 \%$ higher kidney disease burden and less accessibility to ESKD care. Future preventive interventions and special provision for ESKD care to these high-risk groups are necessary. Second, the impact of climate change on kidney health is a pressing issue in the OSEA region [20], and increases in water crisis episodes challenge the sustainability of HD as the main KRT modality [21].

\section{Middle East Asia}

Of the 13 countries comprising the ISN Middle East, 11 participated in the GKHA survey. While most countries, especially those of the Gulf Cooperation Council, are classified as high-income or upper-middle-income groups, existing issues act as barriers in achieving universal health coverage in this region. These issues include geopolitical tensions, refugee crises, hierarchical social structures, and ignorance concerning renal diseases. The CKD and ESKD burden is high in the Middle Eastern region due to high incidence of diabetes mellitus and hypertension, and the burden is expected to increase further [22]. Therefore, health information systems to capture detailed disease epidemiology and national strategies for addressing chronic diseases are needed in this region as only $27 \%$ of the countries in the region have such strategies. Government funding of CKD treatment is high in this region, with two-thirds of countries covering costs of nondialysis CKD medications and all countries covering costs of KRT. HD is the predominant modality of KRT in all countries in the Middle East. This is likely due to lack of funding support and expertise for PD. 
Iran has the highest number of kidney transplantations in the region, 311 per million population, with more than half being deceased donor transplantations. This contrasts with other countries in this region that favor living donor transplantation.

\section{Conclusions}

The GKHA is an important initiative that promotes global, data-driven collaboration to advocate for equitable kidney disease treatment across all countries. The overwhelming response rates from individual countries have facilitated the creation of a platform for information related to kidney diseases. The Asia-Pacific region is heterogeneous in terms of economic development, culture, ethnicity, and epidemiology of diseases. Different countries need to develop strategies specifically for their priorities. International collaboration for scientific and financial inputs is crucial for improving kidney disease care in the emerging economies of the OSEA and North and East Asia regions. These inputs include the increased use of PD and training of the workforce in vascular access interventions. South Asian countries need to focus on preventive interventions to decrease the incidence of CKD and ESKD. Awareness of kidney disease as a complication of chronic diseases and improvement of early community management of communicable diseases likely to cause kidney injury are priorities for preventive intervention. Government spending on healthcare needs an urgent increase in most South Asian countries and a few developing economies of the OSEA to confront the problem of inaccessibility to ex-pensive treatments for kidney failure. Risk mitigation strategies for CKD prevention and research capacity need to be enhanced by the nephrology community in the Middle East.

\section{Conflicts of interest}

All authors have no conflicts of interest to declare.

\section{Authors' contributions}

Conceptualization, Supervision: VJ

Writing-original draft: JB

Writing-review \& editing: JB, VJ

All authors read and approved the final manuscript.

\section{ORCID}

Joyita Bharati, https://orcid.org/0000-0003-1401-8159

Vivekanand Jha, https://orcid.org/0000-0002-8015-9470

\section{References}

1. Liyanage T, Ninomiya T, Jha V, et al. Worldwide access to treatment for end-stage kidney disease: a systematic review. Lancet 2015;385:1975-1982.

2. Ashuntantang G, Osafo C, Olowu WA, et al. Outcomes in adults and children with end-stage kidney disease requiring dialysis in sub-Saharan Africa: a systematic review. Lancet Glob Health 2017;5:e408-e417.

3. Bello AK, Levin A, Lunney M, et al. Global Kidney Health Atlas: a report by the International Society of Nephrology on the Global Burden of End-stage Kidney Disease and Capacity for Kidney Replacement Therapy and Conservative Care across World Countries and Regions. Brussels, Belgium: International Society of Nephrology; 2019.

4. Bharati J, Jha V, Levin A. The Global Kidney Health Atlas: burden and opportunities to improve kidney health worldwide. Ann Nutr Metab 2020;76 Suppl 1:25-30.

5. Bello AK, Levin A, Lunney M, et al. Status of care for end stage kidney disease in countries and regions worldwide: international cross sectional survey. BMJ 2019;367:15873.

6. Okpechi IG, Alrukhaimi M, Ashuntantang GE, et al. Global capacity for clinical research in nephrology: a survey by the International Society of Nephrology. Kidney Int Suppl (2011) 2018;8:82-89

7. Saran R, Robinson B, Abbott KC, et al. US Renal Data System 2017 Annual Data Report: Epidemiology of Kidney Disease in the United States. Am J Kidney Dis 2018;71(3 Suppl 1):A7.

8. Lalji R, Francis A, Wong G, et al. Disparities in end-stage kidney disease care for children: a global survey. Kidney Int 2020;98:527-532.

9. Abraham G, Varughese S, Thandavan T, et al. Chronic kidney disease hotspots in developing countries in South Asia. Clin Kidney J 2016;9:135-141.

10. Ranasinghe P, Mathangasinghe Y, Jayawardena R, Hills AP, Misra A. Prevalence and trends of metabolic syndrome among adults in the asia-pacific region: a systematic review. BMC Public Health 2017;17:101.

11. Zhang L, Wang J, Yang CW, et al. International Society of Nephrology Global Kidney Health Atlas: structures, organization 
and services for the management of kidney failure in North and East Asia. Kidney Int Suppl (2011) 2021;11:e77-e85.

12. Hong YA, Ban TH, Kang CY, et al. Trends in epidemiologic characteristics of end-stage renal disease from 2019 Korean Renal Data System (KORDS). Kidney Res Clin Pract 2021;40:52-61.

13. Zhang L, Long J, Jiang W, et al. Trends in chronic kidney disease in China. NEngl J Med 2016;375:905-906.

14. Jin DC, Yun SR, Lee SW, et al. Lessons from 30 years' data of Korean end-stage renal disease registry, 1985-2015. Kidney Res Clin Pract 2015;34:132-139.

15. Zhang L, Zhao MH, Zuo L, et al. China Kidney Disease Network (CK-NET) 2015 Annual Data Report. Kidney Int Suppl (2011) 2019;9:e1-e81.

16. Divyaveer SS, Ramachandran R, Sahay M, et al. International Society of Nephrology Global Kidney Health Atlas: structures, organization, and services for the management of kidney failure in South Asia. Kidney Int Suppl (2011) 2021;11:e97-e105.

17. Kerr PG, Tran H, Ha Phan HA, et al. Nephrology in the Oceania-South East Asia region: perspectives and challenges. Kidney Int 2018;94:465-470.
18. United States Renal Data System (USRDS). USRDS 2016 annual data report, volume 2: ESRD in the United States [Internet]. Bethesda, MD: USRDS; 2016 [cited 2021 Oct 10]. Available from: https://www.usrds.org/media/1632/v2_c00_intro_16.pdf.

19. Ethier I, Johnson DW, Bello AK, et al. International Society of Nephrology Global Kidney Health Atlas: structures, organization, and services for the management of kidney failure in Oceania and South East Asia. Kidney Int Suppl (2011) 2021;11:e86-e96.

20. Borg M, Bi P, Nitschke M, Williams S, McDonald S. The impact of daily temperature on renal disease incidence: an ecological study. Environ Health 2017;16:114.

21. Thackray L. Water quality and availability concerns in drought for dialysis patients [Internet]. Sidney: Australian Broadcasting Commission; 2019 [cited 2021 Oct 10]. Available from: https://www.abc.net.au/news/2019-07-15/dialysis-concerns-about-the-impact-of-water-shortage/11310952.

22. Mousavi SS, Soleimani A, Mousavi MB. Epidemiology of endstage renal disease in Iran: a review article. Saudi J Kidney Dis Transpl 2014;25:697-702. 